\title{
Clinical and Electrophysiological Differentiation between Periodical and Epileptic Discharges
}

\author{
Vitaliy V. Podlepich ${ }^{1}$ Ivan A. Savin ${ }^{1}$ \\ ${ }^{1}$ Neurocritical Care Unit, Burdenko National Medical Research \\ Center of Neurosurgery, Moscow, Russia
}

J Neuroanaesthesiol Crit Care 2020;7:96-103

\begin{abstract}
Address for correspondence Vitaliy V. Podlepich, Neurocritical Care Unit, Burdenko National Medical Research Center of Neurosurgery, 4th Tverskaya-Yamskaya Street, 16, Moscow 125000, Russia (e-mail: vpodlepich@nsi.ru).
\end{abstract}

\begin{abstract}
Keywords

- Ictal-interictal continuum

- periodic discharges

- nonconvulsive status epilepticus

Clinical differentiation between nonconvulsive status epilepticus (NCSE) and encephalopathy remains challenging. Some patients with encephalopathy exhibit periodic discharges on electroencephalography. In certain cases, however, it is rather difficult to differentiate between epileptic and periodic discharges. The lack of clear differentiation criteria makes it difficult to effectively analyze the studies in this field. In patients with refractory status epilepticus, the wrong diagnosis can lead to insufficient therapy, thereby increasing the frequency of convulsions and the likelihood of a poor outcome. Alternatively, aggressive therapy with antiepileptic drugs and anesthetic agents can aggravate neuronal damage and worsen encephalopathy. In this review, we aimed to determine whether clinical and instrumental methods can be used to differentiate between encephalopathy and epileptic states, to improve the selection of the appropriate treatment strategies. The review considers different approaches to diagnosing encephalopathy and seizures in critically ill patients. In spite of electroencephalography (EEG) still being the main method used to objectively identify brain dysfunction and diagnose epileptic discharges over the past 60 years, improvements in EEG techniques have been accompanied by increase in our understanding of the limitations of electrophysiological methods, leading to the use of additional tools for the differential diagnosis of seizures and encephalopathy. Current studies aim to develop multimodal approaches to the differential diagnosis of encephalopathy and NCSE. Modern reports describe clinical and instrumental approaches for accurately differentiating encephalopathy and epileptic conditions in patients treated in the intensive care unit based on clinical data, EEG, magnetic resonance imaging, positron emission tomography, biochemical analyses, and immune values.
\end{abstract}

\section{Introduction}

Modern approaches to the management of nonconvulsive status epilepticus (NCSE) are effective for cases in which timely diagnosis is followed by targeted anticonvulsant therapy. ${ }^{1}$ However, in some cases, differentiation between NCSE and encephalopathy remains challenging, especially when they exhibit parallel courses, as one may be both a cause and a result of the other. The lack of clear differentiation criteria makes it difficult to effectively analyze the studies in this field since the results are somewhat subjective and unreliable.

Encephalopathy is characterized by temporary or permanent compromises in brain function. ${ }^{2}$ Clinical manifestations of encephalopathy include confusion and decreased level of consciousness or coma. ${ }^{3}$ Patients with encephalopathy can exhibit periodic discharges on electroencephalography $(\mathrm{EEG})^{4}$ ( - Fig. 1). Brain dysfunction can be caused by
DOI https://doi.org/ 10.1055/s-0040-1701590 ISSN 2348-0548.
(C) 2020 Indian Society of Neuroanaesthesiology and Critical Care
License terms

()(1) $\odot \circledast$ 


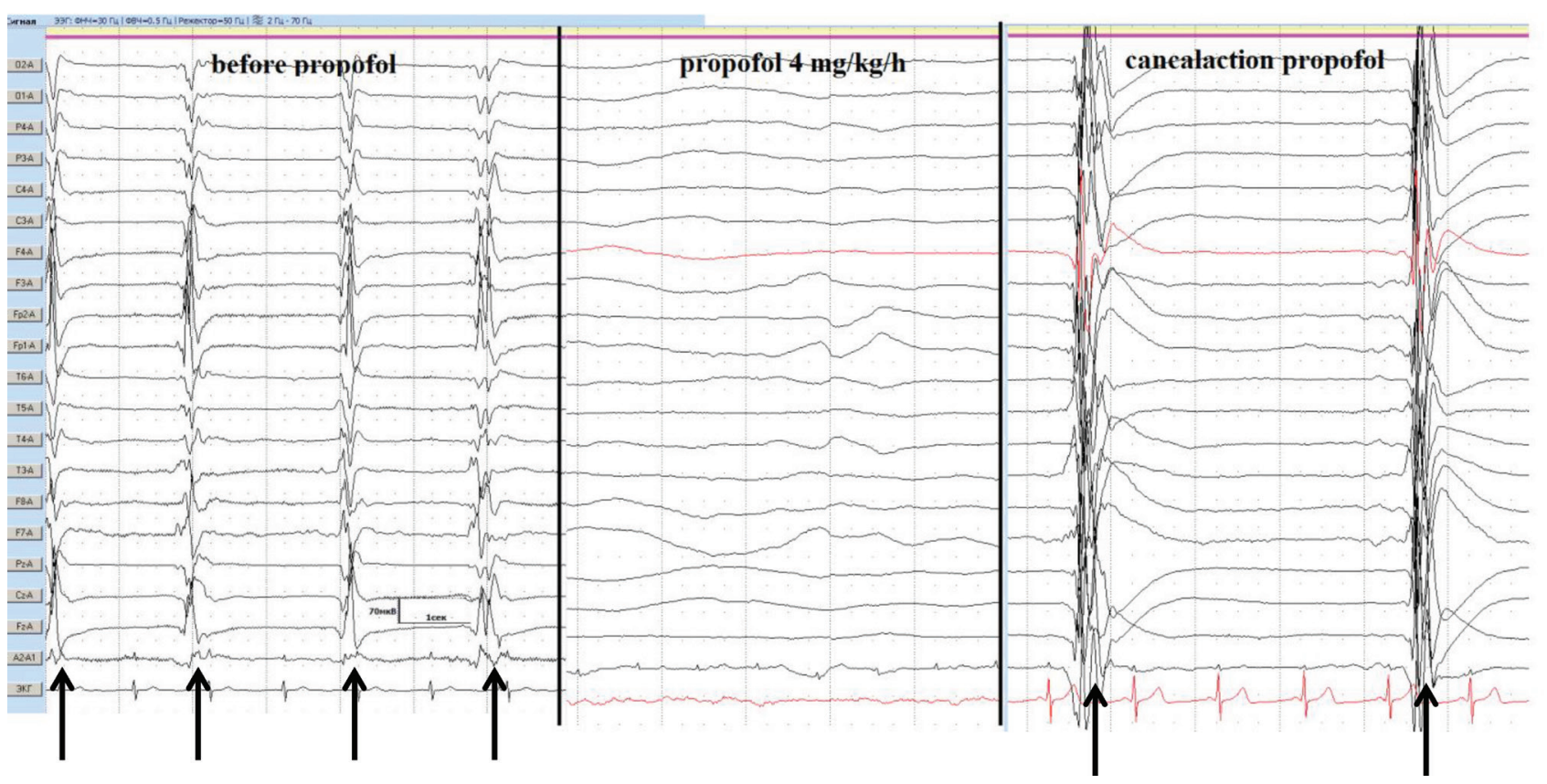

Fig. 1 A 61-year-old woman after CPR with intravenous hypothermia went into coma. Typical GPDs $0.5 \mathrm{~Hz}$ (left third, black arrows) were observed. Her condition was considered as state after global anoxia. The patterns disappeared after taking propofol infusion $4 \mathrm{mg} / \mathrm{kg} / \mathrm{h}$ (middle third). These patterns reverted after withdrawal of propofol infusion (right third, black arrows). Sedation was used for synchronization with mechanical ventilations. CPR, cardiopulmonary resuscitation; GPD, generalized periodic discharge.

various underlying factors such as hypoxia, ${ }^{5-7}$ endocrine ${ }^{8,9}$ and metabolic ${ }^{10}$ disorders, toxicity, ${ }^{11}$ infections, ${ }^{12,13}$ autoimmune diseases, ${ }^{14,15}$ and the effects of status epilepticus (SE). ${ }^{16}$ Addressing the causes of encephalopathy is critical to effective treatment. However, there are currently no specific therapies for treating the consequences of these conditions.

Unlike encephalopathy, NCSE can be attenuated using specific antiepileptic drugs (AEDs) ( - Fig. 2). Clinically, NCSE is also characterized by confusion and a low level of consciousness. In $30 \%$ of cases, SE is associated with complications due to conversion into refractory status epilepticus (RSE). ${ }^{1}$ RSE is treated using a combination of AEDs and continued infusions of anesthetic agents. ${ }^{17}$ The required duration of anesthetic treatment is between 1 and 7 days. Increases in the duration of treatment or number of agents used increases the risk of developing encephalopathy among patients with $\operatorname{RSE}^{18}$ (-Fig. 3).

In certain cases, however, it is rather difficult to differentiate between epileptic and periodic discharges. In patients with RSE, the wrong diagnosis can lead to insufficient therapy, thereby increasing the frequency of convulsions and the likelihood of a poor outcome. Alternatively,

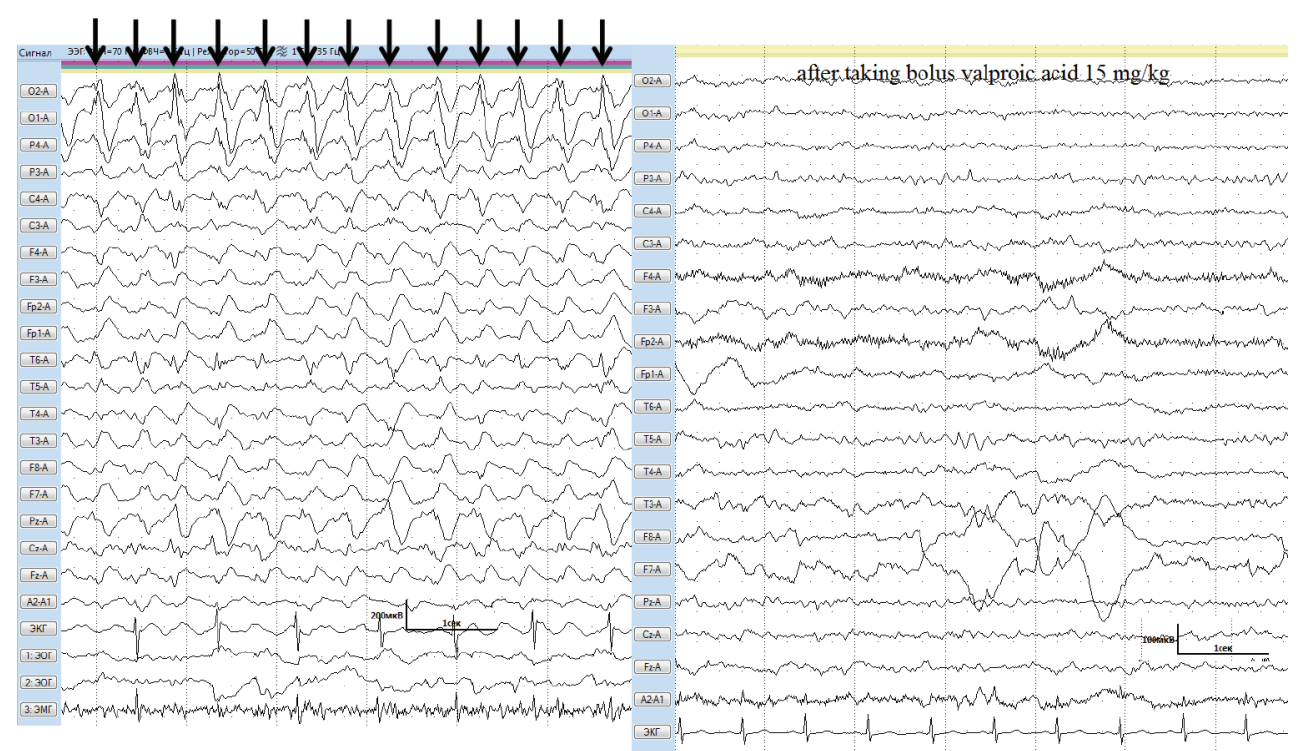

Fig. 2 A 65-year-old woman after removal of frontal meningioma reported confused consciousness and subtle clinical seizures with periodic patterns of $2.5 \mathrm{~Hz}$ (left side, black arrows). Her condition was considered as NCSE. The patterns disappeared after taking bolus valproic acid $15 \mathrm{mg} / \mathrm{kg}$ (right side). The patient was conscious after 2 hours. NCSE, nonconvulsive status epilepticus. 
aggressive therapy with AEDs and anesthetic agents can aggravate neuronal damage and worsen encephalopathy.

The term ictal-interictal continuum (IIC) has been introduced to describe the dynamics of these conditions during continued EEG monitoring. ${ }^{19}$ The IIC includes periodic and epileptic discharges, as well as background activity between them..$^{20}$ In publications, IIC is often referred to as the pattern of uncertainty, which more accurately reflects its dual structure ( - Fig. 4).

In this review, we aimed to determine whether clinical and instrumental methods can be used to differentiate between encephalopathy and epileptic states, to improve the selection of the appropriate treatment strategies. The review considers different approaches to diagnosing encephalopathy and seizures in critically ill patients.

\section{Mechanisms Underlying the Generation of Periodic and Epileptic Discharges}

EEG is the main method used to objectively identify brain dysfunction and diagnose epileptic discharges. For many

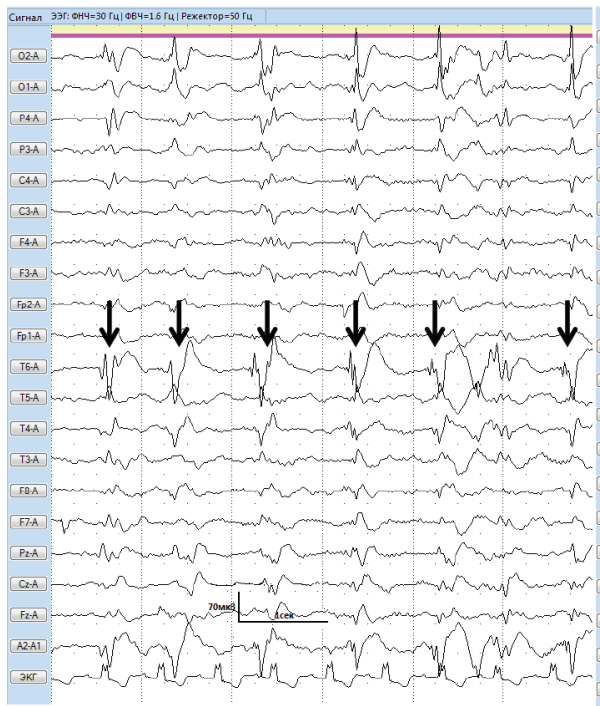

LPD's $1 \mathrm{~Hz}$ in right hemisphere

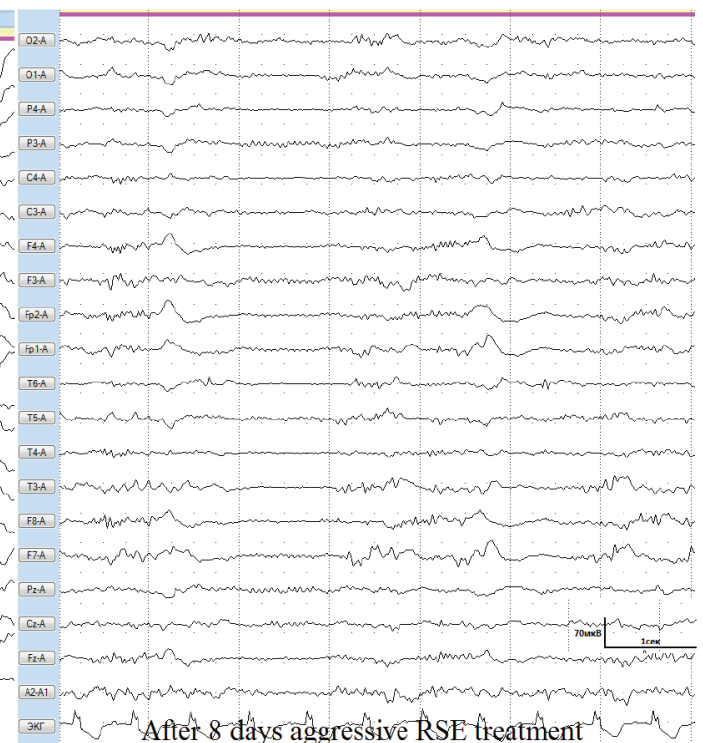

Fig. 3 A 9-year-old child after removal of thalamic teratoma reported electrolytes disorders and went into coma. He had rare subtle clinical seizures and LPDs $(1 \mathrm{~Hz})$ in the right hemisphere (left side, black arrows). His condition was considered as NCSE. Patterns disappeared after 8 days of aggressive RSE treatment (right side). Patient was administered four AEDs and is in a persistent vegetative status. AED, antiepileptic drug; NCSE, nonconvulsive status epilepticus; LPD, lateralized periodic discharges; RSE, refractory status epilepticus.

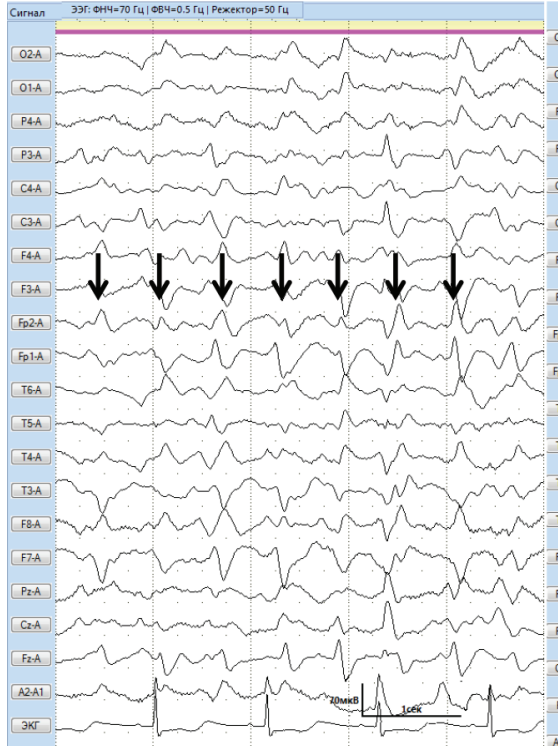

Periodic patterns $2.0 \mathrm{~Hz}$.

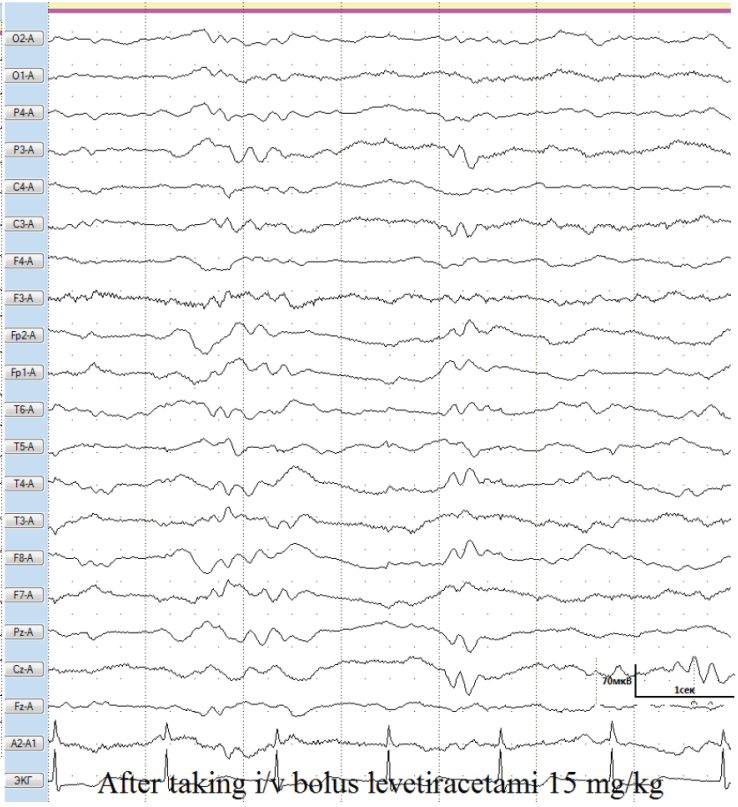

Fig. 4 A 70-year-old man after fossa posterior surgery reported confused consciousness. There are no data about seizures. Periodic patterns of $2.0 \mathrm{~Hz}$ (left side, black arrows) were observed. His condition was considered as possible NCSE. He was administered AEDs. Patterns disappeared after taking intravenous bolus levetiracetam $15 \mathrm{mg} / \mathrm{kg}$ (right side). The patient has the same status. AED, antiepileptic drug; NCSE, nonconvulsive status epilepticus. 
years, researchers believed that rhythmic discharges were related to a disruption in the connections between the cortex and thalamus. ${ }^{21}$ Better neuroimaging methods and new mathematical models have refined this concept. ${ }^{22}$ According to Kalamangalam, rhythmic discharges originate from disruptions in interactions involving the intercolumnar modular cortex..$^{23}$ Deep depolarization of interneurons after electrical stimulation manifest as slow waves. The similar nature of periodic discharges after SE and those caused by infectious or structural brain lesions may be related to the selective vulnerability of interneurons due to repeated depolarization and the relative preservation of pyramidal cells. ${ }^{24}$

These data are in contrast to those from studies in which large excitatory pyramidal cells of the hippocampus became selectively vulnerable during hypoxia, although these cells also registered periodic low-frequency oscillations. ${ }^{25}$

Some studies have reported that periodic discharges result from isolated damage to the subcortical white matter ${ }^{22}$ or subcortical nuclei, ${ }^{26}$ and multifocal brain damage. ${ }^{27}$

These findings highlight that our understanding of the origins of regular high-amplitude, synchronous, low-frequency discharges in the damaged brain is limited, whereas the origins of epileptic discharges have been well described. ${ }^{28-32}$

On the other hand, registration of similar EEG patterns under different clinical conditions can be attributed to multiple mechanisms. More detailed classifications of periodic discharges may aid in comparing these patterns and their morphological substrates.

\section{Classification of Periodic Discharges}

In 2005, the American Association of Electrophysiologists removed the term "epileptiform" from the nomenclature and ruled out the idea that periodic discharges must be associated with epileptic conditions. ${ }^{33}$ In 2012, the conciliation commission American Clinical Neurophysiology Society (ACNS) supplemented and amended the definitions and classification of periodic patterns $\mathrm{s}^{34}$. Their work aimed to accurately separate periodic discharges from other EEG features, including epileptic activity.

Some studies have addressed the problem from a different angle. In 2002, Brenner developed a classification system and criteria for isolating epileptic phenomena from the background EEG. ${ }^{35}$ In 2015, a group of authors led by Trinka achieved separation of epileptic from periodic discharges based on EEG. ${ }^{36}$ However, the main criterion for verification of epileptic discharges was response to treatment with AEDs, which can lead to misdiagnosis of the discharges as periodic in some patients with RES.

Thus, electrophysiological differentiation of periodic and epileptic discharges requires an experienced specialist. Nonetheless, one cannot disregard the relativity of the criteria described. Consequently, this assessment leads to two phenomena:

1. The results of the EEG analysis depend on the specialist's clinical experience.

2. Previously completed studies cannot be evaluated according to the new criteria or compared with new studies.

\section{Epidemiology of Periodic Discharges}

The occurrence rates for periodic discharges vary depending on the criteria chosen by the authors and the characteristics of the patient sample. In retrospective studies, periodic discharges were recorded in 0.2 to $1 \%$ of cases. ${ }^{37-39}$ The low occurrence of periodic discharges in these three studies above is likely due to technical difficulties in recording and the short EEG record utilized. In 2012, Foreman observed generalized periodic discharges (GPDs) in $4.5 \%$ of 200 critically ill patients ${ }^{40}$ In 2013, Gaspard et al observed lateralized periodic discharges (LPDs) and lateralized rhythmic delta activity (LRDA) in $8.6 \%$ and $4.7 \%$ of patients, respectively. In their 2016 multicenter study, Ruiz et al identified periodic discharges in 2,019 of 4,772 patients (42.3\%). ${ }^{41}$ Among them, LPDs accounted for $32 \%$, bilateral periodic discharges (BiPDs) for $5 \%$, GPDs for $27 \%$, and generalized rhythmic delta activity (GRDA) for 36\%. (Here, calculations are performed based on the data provided in the original studies.)

Notably, the last two studies utilized the 2012 Association of American Electrophysiologists guidelines. However, Ruiz et al published data for 493 patients who had both periodic and epileptic discharges. ${ }^{41}$ Epileptic seizures were identified clinically; however, if clinical seizures are absent, the only way to separate periodic and epileptic discharges is via a visual analysis of the EEG. The authors did not specify the frequency of periodic discharges in patients with different nosology. Nevertheless, a clear predominance of patients with primary brain damage was observed in accordance with several previous studies. ${ }^{21,42-45}$

Various authors ${ }^{40,44,46-48}$ have distinguished the following clinical conditions with periodic discharges:

1. Local ischemic brain damage (stroke): 27 to $45 \%$.

2. CNS infections: 6 to $18 \%$.

3. Intracranial hemorrhage: 2 to $17 \%$.

4. Anoxic brain damage: 2 to $14 \%$.

5. Brain tumors: 7 to $11 \%$.

6. Metabolic and toxic disorders: 9 to $55 \%$.

7. Sepsis: $35 \%$.

All these clinical conditions may cause epileptic seizures. ${ }^{1}$ At the same time, Snodgrass et al proposed viewing periodic epileptic discharges as part of SE and a form of "fatigued" clonic seizures (or their termination). ${ }^{46}$ Below, we further consider the relationship between periodic and epileptic discharges within the IIC and NCSE. (We have intentionally used different nomenclature for periodic patterns to emphasize classification used by the original authors.)

\section{Periodic Discharges as a Predictor of Status Epilepticus}

Given some of the shared mechanisms underlying the generation of periodic and epileptic discharges, as well as their clinical similarity and underlying causes, some studies have assessed the likelihood of developing SE during the registration of periodic EEG patterns. While Moralez et al reported that periodic discharges have no prognostic value for 
seizures, ${ }^{47}$ Foreman et al reported that GPDs were associated with the development of NCSE, but not with poor outcomes. ${ }^{40}$ In their sample, most patients had sepsis or metabolic/toxic brain lesions.

Claassen et al investigated patients treated in the neurointensive care unit, observing that electrographic equivalents of seizures frequently occurred in combination with PEDs. Epileptic activity exhibited the strongest association with periodic lateralized epileptiform discharges (PLEDs) ${ }^{49}$ and focal stimulus-induced rhythmic, periodic, or ictal discharges (SIRPIDs). ${ }^{44}$ Viarasilpa et al observed similar findings in patients with acute encephalitis. Although periodic discharges were associated with nonconvulsive epileptic seizures due to LPDs, the authors observed no associations with SIRPIDs, GPDs, BiPDs, or any form of RDA..$^{45}$ A study performed 10 years earlier revealed a link between electrographic seizures and PEDs in general and with PLEDs, GPEDs, and BiPEDs in patients with CNS infections. In a multivariable logistic regression analysis corrected for viral causes and protein levels in the CSF, PEDs were independently associated with status epilepticus. ${ }^{43}$ Despite the similar sample sizes and analytical methods in the last two studies, differences in the results may be due to the use of the latest classification of periodic discharges in the more recent study.

Ruiz et al observed that LPDs, LRDA, GPDs, and BiPDs were associated with seizures in patients treated in the intensive care unit, without correction for frequency or the addition of any of the "plus" modifiers. After correcting for frequency patterns and "plus" modifiers, SE was associated only with LPDs and LRDA plus. ${ }^{41} \mathrm{~A}$ univariate analysis by Koren et al revealed that nonconvulsive epileptic activity or IIC during the first 30 minutes is a strong predictor of NCSE development. In the multiregression analysis, the registration of individual epileptiform discharges or IIC predicted a high risk of developing NCSE. The authors did not state whether certain types of periodic discharges were associated with the risk of NCSE development; nevertheless, among all other patterns, LPDs were registered within the IIC in $65 \%$ of cases. ${ }^{50}$

Thus, most authors regard the registration of periodic discharges on EEG as a predictor of NCSE development. LPDs are more often associated with subsequent seizure development. However, using AEDs is still an open question.

\section{Association of Periodic and Epileptic Discharges with Structural and Functional Changes}

In 2009, Goyal et al identified peri-ictal changes in the MR signal in a significant proportion of patients with SE. These changes were most apparent when magnetic resonance imaging (MRI) was performed immediately after the cessation of SE. Peri-ictal changes in the MR signal may be associated with cytotoxic edema caused by increased energy metabolism, hyperperfusion, impaired $\mathrm{Na} / \mathrm{K}$ ATPase function, and excessive release of stimulating amino acids as a result of ictal activity. ${ }^{51}$ At the same time, several studies have investigated the nature of periodic discharges using neuroimaging techniques.

In several clinical cases it was observed that the dynamics of partial epilepsy transformed into PLEDs and compared with positron emission tomography (PET) data. The authors concluded that the EEG pattern exhibits epilepsia partialis continua. ${ }^{52,53}$

Assal et al observed local increases in cerebral blood flood (CBF) in all patients with PLEDs using single photon emission computed tomography (SPECT). The localization of periodic discharges coincided with PET data in $94 \%$ of participants, ${ }^{54}$ suggesting that epileptic activity is an underlying cause of periodic patterns. However, all patients included in the study had epileptic seizures, which may indirectly reflect the initial ictal nature of the observed patterns.

In 2017, Struck et al compared EEG and PET data in 18 patients. The authors concluded that the hypermetabolism detected by SPECT in patients with IIC is a specific marker for convulsive SE and NCSE. ${ }^{55}$ However, the statistical power of the study was insufficient. Moreover, the criteria for diagnosing status epilepticus were not standardized.

In 2015, Rennebaum et al reported that PLEDs and epileptic discharges were observed much more frequently in patients with peri-ictal restriction diffusion data. It should be noted that the authors did not use the ACNS classification for differentiating between periodic and epileptic discharges, and PLEDs dominated the EEG in $16 \%$ of patients. Patients without peri-ictal hyperintensities on DWI exhibited a greater frequency of bilateral or generalized periodic and epileptic discharges. However, in 35\% of patients, neither EEG nor clinical and anamnestic data could be used to separate SE from severe encephalopathy. ${ }^{56}$ The authors concluded that "local" metabolic abnormalities due to ictal activity contributed to the development of PLEDs, and to peri-ictal hyperintensities on DWI. Moreover, PLED registration in patients with peri-ictal DWI restriction in the thalamic region indicates that corticothalamic fibers are not disconnected, as suggested by Cobb, ${ }^{21}$ but are rather synchronized during SE.

Narayanan evaluated the MRIs of 10 patients with epileptic discharges and LPDs. Peri-ictal DWI restriction was detected in all five patients with epileptic activity. Structural damage was detected in the T2 mode in four of five patients with LPDs. ${ }^{57}$ The limited sample size did not allow the authors to determine statistical significance.

Due to the complexity of comparing EEG and neuroimaging data in critically ill patients, these studies can be used to establish trends, but not to confirm the epileptic nature of periodic discharges. However, in all studies mentioned above, LPDs were regarded as potentially epileptic.

\section{Relationship of Periodic and Epileptic Discharges with Metabolic Changes in the Living Brain}

Studies of local metabolic changes in the living brain have yielded the most indicative model regarding the similarity of the pathophysiological mechanisms underlying periodic and epileptic discharges. Using local measurement techniques, Vespa et al reported that CBF, glucose/oxygen consumption, lactate levels, local brain temperature, and intracranial pressure (ICP) increase during the registration of periodic patterns on EEG. ${ }^{58}$ Similar changes are observed during epileptic activity in patients with traumatic brain injury (TBI).$^{59}$ Katyal et al also 
noted that three-phase waves on EEG can be significant predictors of intracranial hypertension. ${ }^{60}$ Attention should be drawn to the relatively small number of works in this area performed by a single group of researchers.

\section{Periodic Discharges as a Predictor of Outcomes}

According to Claassen et al, the following EEG patterns are considered predictors of poor outcomes in patients with intracerebral hemorrhage (ICH): any PEDs in general and individual PLEDs or SIRPIDs. ${ }^{44}$

Westhall et al analyzed EEG data and other prognostic markers in 134 patients after cardiopulmonary resuscitation (CPR). The authors referred to ACNS criteria, but the design of the study did not allow for unambiguous separation of periodic and epileptic discharges. However, poor outcomes were observed in patients with a high index of regular periodic patterns, in contrast to findings observed in patients with individual epileptic discharges. ${ }^{61}$

In 2016, Alvarez et al reported that background activity and sleep signs on EEG are more reliable predictors of good clinical outcomes after CPR than periodic or epileptic patterns. The regression model included EEG data, clinical information, and etiology. The authors reported that a lack of EEG reactivity was the single feature most closely related to mortality. ${ }^{62}$ In our opinion, the reason for the discrepancies between these results and previous findings may be due to the finding that lack of EEG reactivity is a common symptom among patients with various types of brain damage. Furthermore, this symptom can be observed in patients with a relatively high level of consciousness. Thus, it may be difficult to identify patients with IIC or PDs with spared EEG reactivity.

In 2018, Beretta et al evaluated 166 patients after CPR using systemic hypothermia. The authors reported that careful separation of EEG patterns into epileptic and periodic patterns improves diagnosis, treatment results, and neurological outcomes. ${ }^{42}$ Although this study compared outcomes for GPDs and RSE, which are the most difficult conditions to separate, patients with LPDs were not included.

In a previous study, Japanese authors investigated the prognostic significance of periodic and epileptic discharges, as well as changes on MRI, in patients over 65 with epileptic seizures. In the multiregression analysis, only DWI hyperintensities and RSE were independent prognostic factors for poor outcomes in older adults. ${ }^{63}$ In a subsequent study, the same group of authors demonstrated that in contrast to younger patients, older adults exhibit a higher frequency of LPDs and hyperintensities on DWI associated with SE. Such changes may represent a surrogate marker of neuronal damage due to SE, and the higher incidence of these pathological findings in older adults may reflect the vulnerability of the aging brain. ${ }^{64}$

Although differentiation of epileptic and periodic discharges in the case of NCSE and encephalopathy remains challenging, this allows for the selection of patients likely to respond to complex anticonvulsant therapy, which may improve the chance of good outcomes. Similarly, registration of PDs may reflect severe brain damage, allowing for the selection of patients likely to experience poor outcomes.

\section{Multimodal Approaches to the Differentiation of Periodic and Epileptic Patterns}

Periodic and epileptic patterns involve a continuum of electrographic characteristics (e.g., duration, frequency, and prevalence) and are influenced by numerous physiological factors such as cerebral perfusion and metabolism. Acting together, pathophysiological features associated with periodic discharges can cause secondary neuronal damage, leading to reversible or irreversible neurological disorders. A multimodal approach may allow for the identification of physiological abnormalities that go beyond what can be obtained via EEG alone. Two studies published in 2018 incorporated data obtained over three decades of investigating periodic patterns and their relationships with epileptic activity. ${ }^{65,66}$ These reports describe clinical and instrumental approaches for accurately differentiating encephalopathy and epileptic conditions in patients treated in the intensive care unit based on clinical data, EEG, MRI, PET, biochemical analyses, and immune values. These studies further highlight the role of multimodal approaches in the stratification of individual diagnostic phenotypes.

\section{Conclusion}

The clinical signs of encephalopathy and NCSE are nonspecific, and these two states cannot be differentiated without the use of additional instrumental methods. EEG has been used to differentiate between encephalopathy and NCSE over the past 60 years. Improvements in EEG techniques have been accompanied by increase in our understanding of the limitations of electrophysiological methods, leading to the use of additional tools for the differential diagnosis of seizures and encephalopathy. Such tools include neuroimaging methods, such as MRI and PET, which can be used to assess local metabolism in the living brain, and biochemical markers. Current studies aim to develop multimodal approaches to the differential diagnosis of encephalopathy and NCSE, although further research is required to determine the clinical efficiency of these approaches. To date, it remains impossible to determine whether periodic and epileptic discharges represent a manifestation of one process or are parallel processes of different nature that occur due to a lack of energy in brain cells.

\section{Conflict of Interest}

None declared.

\section{Acknowledgments}

We would like to thank Editage (www.editage.com) for English language editing.

\section{References}

1 Panayiotis N. Varelas, Claassen J. Seizures in Critical Care. A Guide to Diagnosis and Therapeutics. 3rd ed. New York, NY: Humana Press; 2017:103-117

2 The British Medical Association. Illustrated Medical Dictionary BMA. DK: The British Medical Association (BMA); 2013

3 Larner AJ. A Dictionary of Neurological Signs. 4th ed. Cham, Switzerland: Springer; 2016 
4 Faigle R, Sutter R, Kaplan PW. Electroencephalography of encephalopathy in patients with endocrine and metabolic disorders. J Clin Neurophysiol 2013;30(5):505-516

5 Fugate JE. Anoxic-ischemic brain injury. Neurol Clin 2017;35(4):601-611

6 Elmer J, Callaway CW. The brain after cardiac arrest. Semin Neurol 2017;37(1):19-24

7 Rubinos C, Ruland S. Neurologic complications in the intensive care unit. Curr Neurol Neurosci Rep 2016;16(6):57

8 Barake M, Klibanski A, Tritos NA. Management of endocrine disease: impulse control disorders in patients with hyperpolactinemia treated with dopamine agonists-how much should we worry? Eur J Endocrinol 2018;179(6):R287-R296

9 He XY, Dobkin C, Yang SY. 17 $\beta$-hydroxysteroid dehydrogenases and neurosteroid metabolism in the central nervous system. Mol Cell Endocrinol 2019;489:92-97

10 Achinger SG, Ayus JC. Treatment of hyponatremic encephalopathy in the critically ill. Crit Care Med 2017;45(10):1762-1771

11 White J, Weinstein SA, De Haro L, et al. Mushroom poisoning: a proposed new clinical classification. Toxicon 2019;157:53-65

12 Robba C, Crippa IA, Taccone FS. Septic encephalopathy. Curr Neurol Neurosci Rep 2018;18(12):82

13 O'Horo JC, Sampathkumar P. Infections in neurocritical care. Neurocrit Care 2017;27(3):458-467

14 Shan F, Long Y, Qiu W. Autoimmune glial fibrillary acidic protein astrocytopathy: a review of the literature. Front Immunol 2018;9:2802

15 Sahebari M, Rezaieyazdi Z, Khodashahi M, Abbasi B, Ayatollahi F. Brain single photon emission computed tomography scan (spect) and functional MRI in systemic lupus erythematosus patients with cognitive dysfunction: a systematic review. Asia Ocean J Nucl Med Biol 2018;6(2):97-107

16 Fauser S, Tumani H. Epilepsy. Handb Clin Neurol 2017; 146:259-266

17 Brophy GM, Bell R, Claassen J, et al; Neurocritical Care Society Status Epilepticus Guideline Writing Committee. Guidelines for the evaluation and management of status epilepticus. Neurocrit Care 2012;17(1):3-23

18 Andresen JM, Girard TD, Pandharipande PP, Davidson MA, Ely EW, Watson PL. Burst suppression on processed electroencephalography as a predictor of postcoma delirium in mechanically ventilated ICU patients. Crit Care Med 2014;42(10):2244-2251

19 Pohlmann-Eden B, Hoch DB, Cochius JI, Chiappa KH. Periodic lateralized epileptiform discharges-a critical review. J Clin Neurophysiol 1996;13(6):519-530

20 Chong DJ, Hirsch LJ. Which EEG patterns warrant treatment in the critically ill? Reviewing the evidence for treatment of periodic epileptiform discharges and related patterns. J Clin Neurophysiol 2005;22(2):79-91

21 Cobb W, Hill D. Electroencephalogram in subacute progressive encephalitis. Brain 1950;73(3):392-404

22 Raroque HG Jr, Purdy P. Lesion localization in periodic lateralized epileptiform discharges: gray or white matter. Epilepsia 1995;36(1):58-62

23 Kalamangalam GP, Slater JD. Periodic lateralized epileptiform discharges and afterdischarges: common dynamic mechanisms. J Clin Neurophysiol 2015;32(4):331-340

24 Kalamangalam GP, Tandon N, Slater JD. Dynamic mechanisms underlying afterdischarge: a human subdural recording study. Clin Neurophysiol 2014;125(7):1324-1338

25 Howarth C, Gleeson P, Attwell D. Updated energy budgets for neural computation in the neocortex and cerebellum. J Cereb Blood Flow Metab 2012;32(7):1222-1232

26 Gross DW, Quesney LF, Sadikot AF. Chronic periodic lateralized epileptiform discharges during sleep in a patient with caudate nucleus atrophy: insights into the anatomical circuitry of PLEDs. Electroencephalogr Clin Neurophysiol 1998;107(6):434-438

27 Fishman O, Legatt AD. PLEDs following control of seizures and at the end of life. Clin EEG Neurosci 2010;41(1):11-14

28 Wyler AR, Ojemann GA, Ward AA Jr. Neurons in human epileptic cortex: correlation between unit and EEG activity. Ann Neurol 1982;11(3):301-308

29 Trinka E, Brigo F, Shorvon S. Recent advances in status epilepticus. Curr Opin Neurol 2016;29(2):189-198

30 Bate L, Gardiner M. Molecular genetics of human epilepsies. Expert Rev Mol Med 1999;1999:1-22

31 Naylor DE, Liu H, Wasterlain CG. Trafficking of GABA(A) receptors, loss of inhibition, and a mechanism for pharmacoresistance in status epilepticus. J Neurosci 2005;25(34):7724-7733

32 Avanzini G, Franceschetti S, Avoni P, Liguori R. Molecular biology of channelopathies: impact on diagnosis and treatment. Expert Rev Neurother 2004;4(3):519-539

33 Hirsch LJ, Brenner RP, Drislane FW, et al. The ACNS subcommittee on research terminology for continuous EEG monitoring: proposed standardized terminology for rhythmic and periodic EEG patterns encountered in critically ill patients. J Clin Neurophysiol 2005;22(2):128-135

34 Hirsch LJ, LaRoche SM, Gaspard N, et al. American Clinical Neurophysiology Society's Standardized Critical Care EEG Terminology: 2012 version. J Clin Neurophysiol 2013;30(1):1-27

35 Brenner RP. Is it status? Epilepsia 2002;43(Suppl 3):103-113

36 Trinka E, Leitinger M. Which EEG patterns in coma are nonconvulsive status epilepticus? Epilepsy Behav 2015;49:203-222

37 Kuroiwa Y, Celesia GG. Clinical significance of periodic EEG patterns. Arch Neurol 1980;37(1):15-20

38 Yemisci M, Gurer G, Saygi S, Ciger A. Generalised periodic epileptiform discharges: clinical features, neuroradiological evaluation and prognosis in 37 adult patients. Seizure 2003;12(7):465-472

39 San-Juan OD, Chiappa KH, Costello DJ, Cole AJ. Periodic epileptiform discharges in hypoxic encephalopathy: BiPLEDs and GPEDs as a poor prognosis for survival. Seizure 2009;18(5):365-368

40 Foreman B, Claassen J, Abou Khaled K, et al. Generalized periodic discharges in the critically ill: a case-control study of 200 patients. Neurology 2012;79(19):1951-1960

41 Rodriguez Ruiz A, Vlachy J, Lee JW, et al; Critical Care EEG Monitoring Research Consortium. Association of periodic and rhythmic electroencephalographic patterns with seizures in critically ill patients. JAMA Neurol 2017;74(2):181-188

42 Beretta S, Coppo A, Bianchi E, et al. Neurologic outcome of postanoxic refractory status epilepticus after aggressive treatment. Neurology 2018;91(23):e2153-e2162

43 Carrera E, Claassen J, Oddo M, Emerson RG, Mayer SA, Hirsch LJ. Continuous electroencephalographic monitoring in critically ill patients with central nervous system infections. Arch Neurol 2008;65(12):1612-1618

44 Claassen J, Jetté N, Chum F, et al. Electrographic seizures and periodic discharges after intracerebral hemorrhage. Neurology 2007;69(13):1356-1365

45 Viarasilpa T, Panyavachiraporn N, Osman G, et al. Electrographic seizures in patients with acute encephalitis. Neurocrit Care 2019;30(1):207-215

46 Snodgrass SM, Tsuburaya K, Ajmone-Marsan C. Clinical significance of periodic lateralized epileptiform discharges: relationship with status epilepticus. J Clin Neurophysiol 1989;6(2):159-172

47 García-Morales I, García MT, Galán-Dávila L, et al. Periodic lateralized epileptiform discharges: etiology, clinical aspects, seizures, and evolution in 130 patients. J Clin Neurophysiol 2002;19(2):172-177 
48 Orta DS, Chiappa KH, Quiroz AZ, Costello DJ, Cole AJ. Prognostic implications of periodic epileptiform discharges. Arch Neurol 2009;66(8):985-991

49 Claassen J, Mayer SA, Kowalski RG, Emerson RG, Hirsch LJ. Detection of electrographic seizures with continuous EEG monitoring in critically ill patients. Neurology 2004;62(10):1743-1748

50 Koren J, Herta J, Draschtak S, et al. Early epileptiform discharges and clinical signs predict nonconvulsive status epilepticus on continuous EEG. Neurocrit Care 2018;29(3):388-395

51 Goyal MK, Sinha S, Ravishankar S, Shivshankar JJ. Peri-ictal signal changes in seven patients with status epilepticus: interesting MRI observations. Neuroradiology 2009;51(3):151-161

52 Bozkurt MF, Saygi S, Erbas B. SPECT in a patient with postictal PLEDs: is hyperperfusion evidence of electrical seizure? Clin Electroencephalogr 2002;33(4):171-173

53 Handforth A, Cheng JT, Mandelkern MA, Treiman DM. Markedly increased mesiotemporal lobe metabolism in a case with PLEDs: further evidence that PLEDs are a manifestation of partial status epilepticus. Epilepsia 1994;35(4):876-881

54 Assal F, Papazyan JP, Slosman DO, Jallon P, Goerres GW. SPECT in periodic lateralized epileptiform discharges (PLEDs): a form of partial status epilepticus? Seizure 2001;10(4):260-265

55 Struck AF, Westover MB, Hall LT, Deck GM, Cole AJ, Rosenthal ES. Metabolic correlates of the ictal-interictal continuum: FDG-PET during continuous EEG. Neurocrit Care 2016;24(3):324-331

56 Rennebaum F, Kassubek J, Pinkhardt E, et al. Status epilepticus: clinical characteristics and EEG patterns associated with and without MRI diffusion restriction in 69 patients. Epilepsy Res 2016;120:55-64

57 Narayanan J. Can diffusion-weighted imaging be used as a tool to predict seizures in patients with PLEDS? Epileptic Disord 2016;18(4):440-446
58 Vespa P, Tubi M, Claassen J, et al. Metabolic crisis occurs with seizures and periodic discharges after brain trauma. Ann Neurol 2016;79(4):579-590

59 Vespa PM, Miller C, McArthur D, et al. Nonconvulsive electrographic seizures after traumatic brain injury result in a delayed, prolonged increase in intracranial pressure and metabolic crisis. Crit Care Med 2007;35(12):2830-2836

60 Katyal N, Sarwal A, George P, Banik B, Newey CR. The relationship of triphasic waves with intracranial pressure as a possible prognostic marker in traumatic brain injury. Case Rep Neurol Med 2017;2017:4742026

61 Westhall E, Rosén I, Rundgren M, et al. Time to epileptiform activity and EEG background recovery are independent predictors after cardiac arrest. Clin Neurophysiol 2018;129(8):1660-1668

62 Alvarez V, Drislane FW, Westover MB, Dworetzky BA, Lee JW. Characteristics and role in outcome prediction of continuous EEG after status epilepticus: a prospective observational cohort. Epilepsia 2015;56(6):933-941

63 Yoshimura H, Matsumoto R, Ueda H, et al. Status epilepticus in the elderly: prognostic implications of rhythmic and periodic patterns in electroencephalography and hyperintensities on diffusion-weighted imaging. J Neurol Sci 2016;370:284-289

64 Yoshimura $\mathrm{H}$, Matsumoto $\mathrm{R}$, Ueda $\mathrm{H}$, et al. Status epilepticus in the elderly: comparison with younger adults in a comprehensive community hospital. Seizure 2018;61:23-29

65 Bauerschmidt A, Rubinos C, Claassen J. Approach to managing periodic discharges. J Clin Neurophysiol 2018;35(4):309-313

66 Kapinos G, Trinka E, Kaplan PW. Multimodal approach to decision to treat critically ill patients with periodic or rhythmic patterns using an ictal-interictal continuum spectral severity score. J Clin Neurophysiol 2018;35(4):314-324 